(C) The Authors 2015 This is an Open Access article, distributed under the terms of the Creative

Commons Attribution licence (http://creativecommons.org/licenses/by/3.0/), which permits unrestricted

re-use, distribution, and reproduction in any medium, provided the original work is properly cited.

\title{
The cross-sectional association between snacking behaviour and measures of adiposity: the Fenland Study, UK
}

\author{
Laura O'Connor ${ }^{1}$, Soren Brage ${ }^{1}$, Simon J. Griffin ${ }^{1,2}$, Nicholas J. Wareham ${ }^{1}$ and Nita G. Forouhi ${ }^{1 *}$ \\ ${ }^{1}$ MRC Epidemiology Unit, School of Clinical Medicine, Institute of Metabolic Science, University of Cambridge, Cambridge \\ Biomedical Campus, Cambridge CB2 OQQ, UK \\ ${ }^{2}$ Primary Care Unit, Department of Public Health and Primary Care, School of Clinical Medicine, Institute of Public Health, \\ University of Cambridge, Cambridge Biomedical Campus, Cambridge CB2 OQQ, UK
}

(Submitted 8 January 2015 - Final revision received 27 May 2015 - Accepted 22 June 2015 - First published online 7 September 2015)

\section{Abstract}

Unhealthy dietary behaviours may contribute to obesity along with energy imbalance. Both positive and null associations of snacking and BMI have been reported, but the association between snacking and total adiposity or pattern of fat deposition remains unevaluated. The objective of this study was to investigate the associations between snacking frequency and detailed adiposity measurements. A total of 10092 adults residing in Cambridgeshire, England, self-completed eating pattern snacking frequency, FFQ and physical activity questionnaires. Measurements included anthropometry, body composition using dual-energy X-ray absorptiometry scan and ultrasound and assessment of physical activity energy expenditure using heart rate and movement sensing. Linear regression analyses were conducted adjusted for age, socio-demographics, dietary quality, energy intake, PAEE and screen time by sex and BMI status. Among normal-weight individuals (BMI $<25 \mathrm{~kg} / \mathrm{m}^{2}$ ), each additional snack was inversely associated with obesity measures: lower total body fat in men and women $(-0.41(95 \% \mathrm{CI}-0 \cdot 74,-0 \cdot 07) \%,-0 \cdot 41(-0 \cdot 67,-0 \cdot 15) \%$, respectively) and waist circumference $(-0.52(-0.90,-0.14) \mathrm{cm})$ in men. In contrast, among the overweight/obese $\left(\mathrm{BMI} \geq 25 \mathrm{~kg} / \mathrm{m}^{2}\right)$, there were positive associations: higher waist circumference $(0.80(0.34,0.28) \mathrm{cm})$ and subcutaneous fat $(0.06(0.01,0.110) \mathrm{cm})$ in women and waist circumference $(0.37(0.00,0.73) \mathrm{cm})$ in men. Comparing intakes of snack-type foods showed that participants with $\mathrm{BMI} \geq 25 \mathrm{~kg} / \mathrm{m}^{2}$ had higher intakes of crisps, sweets, chocolates and ice-creams and lower intakes of yoghurt and nuts compared with normal-weight participants. Adjusting for these foods in a model that included a BMI-snacking interaction term attenuated all the associations to null. Snacking frequency may be associated with higher or lower adiposity, with the direction of association being differential by BMI status and dependent on snack food choice. Improving snack choices could contribute to anti-obesity public health interventions

Key words: Body composition: Adiposity: Obesity: Snacking: Eating patterns

Eating patterns may contribute to the obesity epidemic along with the effects of energy imbalance. In particular, a shift in eating patterns away from the three-meals-a-day model and towards more of a 'grazing pattern'(1) and an increase in snacking in recent years ${ }^{(2)}$ have been proposed to promote obesity $^{(3)}$. The prevalence of snacking among adults in the USA increased from 71 to $97 \%$ between 1997/1998 and 2003/2006, and the contribution of snacks to energy intake increased from 18 to $24 \%^{(1)}$. Equivalent information on adults in the UK has not been published thus far.

Early investigations often reported an inverse association between eating frequency and adiposity, which was later attributed to reporting bias and the effects of post boc changes in dietary patterns as a consequence of weight gain ${ }^{(4)}$. However, snacking frequency may nonetheless be associated with total adiposity or a pattern of fat deposition, as thus far inconsistent findings, both positive ${ }^{(5,6)}$ and null ${ }^{(7,8)}$, for an association between snacking frequency and BMI, as the only measure of adiposity, have been reported in adults.

Investigating snacking behaviour has many challenges. First, there is lack of an agreed definition of which meals constitute a snack. Second, there is ambiguity in the terminology as snacking frequency has not been distinguished from consumption of snack foods. Third, snacking frequency has been associated with increases in both 'healthy' and 'unhealthy' food choices ${ }^{(9)}$. Furthermore, physical activity (PA) may confound the associations between snacking and adiposity - for example, sedentary behaviour has been positively associated with consumption of energy-dense snacks and inversely associated with fruit and vegetable consumption $^{(10)}$, both of which may be consumed as snacks.

The aim of this study was to investigate the association between self-defined snacking frequency and adiposity measures among adult men and women.

Abbreviations: DEXA, dual-energy X-ray absorptiometry; PA, physical activity; PAEE, physical activity energy expenditure.

* Corresponding author: N. Forouhi, email nita.forouhi@epid.cam.ac.uk 


\section{Methods}

\section{Study population}

The Fenland Study is an on-going population-based study designed to investigate the interactions between genetic and lifestyle factors on the risk of obesity and related metabolic traits. Volunteers born between 1950 and 1975 were recruited from general practice lists in and around Cambridgeshire in the East of England. Recruitment began in 2005, and data were available on 10452 participants at the time of analyses, with a response rate of $27 \%$.

Exclusion criteria included prevalent diabetes, pregnancy or lactation, inability to walk unaided, psychosis or terminal illness. All volunteers gave their written informed consent, and the study was approved by the Cambridge Local Research Ethics Committee.

Participants were invited to attend one of the three testing sites (Ely, Wisbech or Cambridge) for a single visit, where on the test day they completed a number of questionnaires and assessments as detailed below.

\section{Exposure: snacking frequency}

Participants completed an eating pattern questionnaire in which they were asked to describe the meals or snacks that they usually eat during a 24-h period, using a grid that was divided into 2-h slots and offered four choices of meal type: main meal (e.g. meat with potatoes, pizza, lasagne, fish and chips, burgers, fried breakfast), light meal (e.g. porridge, cereal, toast, sandwiches, soup, salad, omelette), snack (e.g. biscuits, cake, fruit, sweets, chocolate, crisps, nuts, ice-cream) and drink-only snack (e.g. drinks with some milk or sugar in; not 'low-calorie' drinks or water). No instruction was given as to whether the 24-h period should be a weekday or a weekend day. Participants were given the option of choosing more than one meal type per time slot. Snacking frequency was calculated as the frequency of food-only snacks that the participant reported. Drink-only snacks were not included due to suggested differences in the satiating effects of foods and drinks ${ }^{(11)}$. The analyses were adjusted for frequency of consumption of other meal types to assess the independent association of snacking frequency and measures of adiposity.

\section{Outcomes: anthropometric and body composition measurements}

At the test site facility, weight and height were measured barefoot and wearing light clothing using standardised procedures. BMI was calculated as weight $(\mathrm{kg})$ divided by the square of height $\left(\mathrm{m}^{2}\right)$. Waist circumference was measured midpoint between the lowest rib margin and the iliac crest to the nearest $0 \cdot 1 \mathrm{~cm}$ with a non-stretchable, fibre-glass insertion tape (D-loop tape; Chasmors Ltd).

Body composition was assessed using dual-energy X-ray absorptiometry (DEXA; Lunar Prodigy Advanced fan beam scanner (GE Healthcare)) and ultrasonography (LOGIQ e ultrasound system (GE Healthcare)), and has been described in detail elsewhere. ${ }^{(12,13)}$ Percentage total body fat was estimated from DEXA using a three-compartment model (fat mass, fat-free mass and bone mineral mass). Visceral and subcutaneous abdominal fat thicknesses $(\mathrm{cm})$ were determined using ultrasound.

\section{Covariates}

Demographic and lifestyle variables were collected using a selfadministered questionnaire.

\section{Dietary covariates}

Self-reported habitual dietary intakes over the previous year were estimated using a validated 130-item semi-quantitative $\mathrm{FFQ}^{(14)}$. Participants were asked to report the frequency of consumption of a 'medium serving' on a nine-point scale from 'never or once per month' to 'more than six times per d' and were asked to complete supplementary questions on milk, breakfast cereal and the type of fat used for baking and frying. Food intake frequency was converted to food (g/d), energy and nutrient intakes using the FFQ EPIC Tool for Analysis ${ }^{(15)}$. Foods that could conceivably be consumed as snacks (snack-type foods) were identified from the FFQ and it included fruits, vegetables, yoghurts, nuts, crisps, cakes, biscuits, chocolate, ice-cream and sweets. These were included as covariates in an attempt to rule out confounding by food type in the association between snacking frequency and adiposity.

Plasma vitamin $\mathrm{C}(\mu \mathrm{mol} / \mathrm{l})$, an objective marker of fruit and vegetable intake ${ }^{(16)}$, was used here as an indicator of dietary quality in line with the inclusion of promoting fruit and vegetable consumption in food-based dietary guidelines ${ }^{(17,18)}$. Plasma vitamin C levels were assessed from fasting blood samples collected into heparin-containing tubes and stabilised with metaphosphoric acid (10\%) and measured by fluorometric assay within 2 months.

\section{Physical activity and sedentary behaviour covariates}

PA was objectively assessed over $6 \mathrm{~d}$ using a combined heart rate and movement sensor ${ }^{(19)}$, with individual calibration of heart rate performed using a treadmill test ${ }^{(20)}$. Data from free-living were pre-processed ${ }^{(21)}$ and modelled using a branched equation framework ${ }^{(22)}$ to estimate intensity timeseries, which were summarised over time as daily Physical physical activity energy expenditure (PAEE) ( $\mathrm{kJ} / \mathrm{kg}$ per $\mathrm{d}$ ).

A validated questionnaire, the Recent Physical Activity Questionnaire $^{(23)}$, was used to assess usual total PA in the previous 4 weeks. From this, we assessed screen time (hours of TV or video watched per day) as a measure of sedentariness.

\section{Statistical analysis}

After the exclusion of participants who had not completed the 24-h eating pattern questionnaire ( $n$ 339) and those who reported no usual eating occasions ( $n$ 21), data from 10092 participants remained available for analysis. Participants with missing data were retained for analyses; missing categorical data (marital status, $n$ 2826; intentional dieting, $n$ 2500) were coded as missing, and for missing continuous data (alcohol consumption, $n$ 175; years of education, $n$ 210) the cohort median value was applied. 
Analyses were performed using Stata (version 13; Stata Corp.).

Population characteristics by snacking frequency are presented as mean values (standard deviations) or medians (interquartile ranges) for continuous variables and as numbers (percentages) for categorical variables, and differences by frequency of snacking were examined using ANOVA or a Kruskal-Wallis test for difference or the $\chi^{2}$ test for heterogeneity.

Associations between snacking frequency (per unit difference) and body composition were examined using multiple linear regression models. Analyses stratified by sex were conducted a priori because previous literature suggested different snacking behaviours by $\operatorname{sex}^{(9,24)}$ and current analyses showing significant interaction between sex and snacking frequency on body composition parameters $(P<0.05)$. A pragmatic approach was used to account for potential confounders including demographic, lifestyle, social, dietary and PA factors. Model 1 was adjusted for age (years), smoking status (never, former and current), alcohol intake (units/d), age at completion of full-time education (years), test site (Cambridge, Ely, Wisbech) and other eating occasions (frequency/d); model 2 was further adjusted for energy intake $(\mathrm{MJ} / \mathrm{d})$, plasma vitamin $\mathrm{C}(\mu \mathrm{mol} / \mathrm{l})$ as a marker of fruit and vegetable intake providing an objectively measured indication of dietary quality, PAEE $(\mathrm{kJ} / \mathrm{kg}$ per $\mathrm{d})$ and screen time. Occupational social class (routine/manual, intermediate/higher managerial and administrative/professional occupations), marital status (single, married, widowed, separated or divorced) and household income level $(<£ 20000, £ 20000-£ 40000$ or $>£ 40000)$ were also considered but were not significant when entered into the model.

A priori, we tested for interaction between snacking frequency and each of BMI $\left(<25\right.$ or $\left.\geq 25 \mathrm{~kg} / \mathrm{m}^{2}\right)$ and PAEE (< or $\geq$ median: $51.7 \mathrm{~kJ} / \mathrm{kg}$ per $\mathrm{d}$ ), in relation to body composition measures.

\section{Sensitivity analyses}

BMR was estimated using Schofield's equations ${ }^{(25)}$. The ratio of energy intake to BMR (EI:BMR) was calculated for each individual. Those with an EI:BMR ratio of $<1.14$ were classified as under-reporters of energy intake according to cut-off limits developed by Goldberg et al. ${ }^{(26)}$. Regression analyses were repeated excluding those who under-reported energy intake.

To investigate the potential effect of intentional dieting on associations with snacking frequency, we compared findings of the analysis of participants who self-reported being on a weight-loss diet with those who reported not being on a diet.

\section{Results \\ Characteristics}

Among all, $84 \%$ of the women and $75 \%$ of the men reported snacking at least once per day (range $=0-12$ times/d). Women reported higher snacking frequency than men (Table 1). Individuals with higher snacking frequency exhibited greater total eating occasions, frequency of other eating occasions and were younger and had higher PAEE compared with their lower snacking frequency counterparts. Higher snacking frequency was associated with lower social class, shorter education duration, lower alcohol intake and being an ex-smoker.

\section{Snacking frequency and adiposity}

In the adjusted analyses, among women, snacking was positively associated with BMI, waist circumference and visceral and subcutaneous abdominal fat thickness, and was inversely associated with body fat percentage in men (Table 2). Further adjustment (model 2) attenuated the association with body fat percentage in men to null.

There was no significant interaction between snacking frequency and PAEE $(P<0 \cdot 05)$ on any body composition measure in men or women. There was, however, a significant interaction between snacking frequency and BMI on waist circumference, body fat percentage and subcutaneous abdominal fat in both men and women (all $P<0.001$ ) and on visceral fat in women only $(P=0 \cdot 002)$.

Stratified analysis was conducted where an interaction was significant, using model 2. Among normal-weight individuals $\left(\mathrm{BMI}<25 \mathrm{~kg} / \mathrm{m}^{2}\right)$, there was an inverse association between snacking and body fat percentage in both sexes and between snacking and waist circumference in men (Fig. 1). In contrast, among the overweight or obese subjects (BMI $\geq 25 \mathrm{~kg} / \mathrm{m}^{2}$ ), there was a positive association between snacking frequency and waist circumference and subcutaneous fat thickness in women and with waist circumference in men (Fig. 1).

\section{Snack-type foods}

Given the interaction between snacking frequency and BMI, we also examined the differences in the intake of snack-type foods (derived from the FFQ) by BMI status using $t$ test or the Mann-Whitney $U$ test, as appropriate. Those who were overweight or obese had higher intakes of crisps, chocolates, ice-cream and sweets and lower intakes of yoghurt and nuts compared with their normal-weight counterparts $(P<0.05)$ (Table 3).

Moreover, to account for possible confounding by the type of food consumed as a snack, we additionally adjusted the stratified analysis for intakes of snack-type foods. This adjustment did not appreciably change the associations; however, when intakes of snack-type foods were added to the unstratified models, which included a BMI $\times$ snacking frequency interaction term, all associations were attenuated to null (results not shown).

\section{Sensitivity analyses}

Exclusion of those categorised as probable energy underreporters ( $n$ 4661) did not appreciably change the direction, size or statistical significance of the observed associations, nor did any of the sensitivity analyses. Among those intentionally dieting ( $12 \%$ of women and $3 \%$ of men), there were no significant associations between snacking frequency and body composition measures, whereas associations among men and 
Table 1. Characteristics of participants by frequency of snacking*: the Fenland Study, UK ( $n$ 10 092)

(Mean values and standard deviations; medians and interquartile ranges (IQR); numbers and percentages)

\begin{tabular}{|c|c|c|c|c|c|c|c|c|c|c|c|}
\hline \multirow[t]{2}{*}{ Snacking (frequency/d...) } & \multicolumn{2}{|c|}{$0(n 2040)$} & \multicolumn{2}{|c|}{$1($ (n 3921) } & \multicolumn{2}{|c|}{$2(n 2710)$} & \multicolumn{2}{|c|}{$3(n$ 1047) } & \multicolumn{2}{|c|}{$4+(n 374)$} & \multirow[b]{2}{*}{$P+$} \\
\hline & Mean & SD & Mean & SD & Mean & SD & Mean & SD & Mean & SD & \\
\hline Age (years) & 49.5 & $7 \cdot 2$ & $48 \cdot 2$ & $7 \cdot 4$ & $46 \cdot 9$ & $7 \cdot 3$ & $46 \cdot 2$ & $7 \cdot 2$ & $46 \cdot 0$ & $7 \cdot 0$ & $<0.001$ \\
\hline Main meal (frequency/d) & $1 \cdot 1$ & 0.4 & $1 \cdot 1$ & 0.3 & $1 \cdot 1$ & 0.4 & $1 \cdot 1$ & 0.4 & $1 \cdot 2$ & 0.7 & $<0.001$ \\
\hline Light meal (frequency/d) & $1 \cdot 7$ & 0.7 & 1.6 & 0.6 & 1.7 & 0.7 & $1 \cdot 8$ & 0.6 & 1.9 & 0.9 & $<0.001$ \\
\hline Energy-containing drink-only snack (frequency/d) & 2.9 & $2 \cdot 3$ & 3.0 & $2 \cdot 3$ & $3 \cdot 1$ & $2 \cdot 3$ & $3 \cdot 2$ & $2 \cdot 4$ & 3.7 & 2.9 & $<0.001$ \\
\hline Total eating occasions (frequency/d) $\ddagger$ & $5 \cdot 7$ & $2 \cdot 3$ & $6 \cdot 7$ & $2 \cdot 3$ & 8.0 & 2.4 & $9 \cdot 1$ & 2.5 & $11 \cdot 3$ & 3.7 & $<0.001$ \\
\hline BMI $\left(\mathrm{kg} / \mathrm{m}^{2}\right)$ & $26 \cdot 9$ & 4.5 & $26 \cdot 9$ & 4.7 & $26 \cdot 8$ & $5 \cdot 0$ & $26 \cdot 7$ & $5 \cdot 1$ & $26 \cdot 7$ & $5 \cdot 1$ & 0.650 \\
\hline Waist circumference $(\mathrm{cm})$ & $92 \cdot 2$ & $13 \cdot 0$ & $91 \cdot 2$ & 13.4 & 89.9 & $13 \cdot 7$ & $89 \cdot 5$ & $13 \cdot 3$ & $90 \cdot 1$ & $13 \cdot 7$ & $<0.001$ \\
\hline Total body fat (\%) & $32 \cdot 1$ & $8 \cdot 8$ & 32.9 & 8.9 & $33 \cdot 6$ & 9.2 & 33.4 & 9.6 & $32 \cdot 7$ & $10 \cdot 2$ & $<0.001$ \\
\hline Visceral fat thickness (cm) & $5 \cdot 5$ & $2 \cdot 2$ & 5.4 & $2 \cdot 2$ & $5 \cdot 1$ & $2 \cdot 2$ & $5 \cdot 0$ & $2 \cdot 0$ & $5 \cdot 2$ & $2 \cdot 1$ & $<0.001$ \\
\hline Subcutaneous abdominal fat thickness $(\mathrm{cm})$ & $2 \cdot 8$ & $1 \cdot 2$ & 2.9 & $1 \cdot 2$ & 2.9 & $1 \cdot 2$ & 2.9 & $1 \cdot 2$ & 2.9 & $1 \cdot 3$ & 0.002 \\
\hline Physical activity energy expenditure (kJ/kg per d) & $53 \cdot 1$ & $22 \cdot 1$ & 53.9 & $22 \cdot 3$ & $55 \cdot 3$ & 21.9 & $56 \cdot 3$ & 23.5 & $57 \cdot 1$ & 23.4 & $<0.001$ \\
\hline \multirow[t]{2}{*}{ Age at end of full-time education (years)§ } & $18 \cdot 8$ & 4.6 & $18 \cdot 6$ & 4.4 & $18 \cdot 8$ & $4 \cdot 3$ & $19 \cdot 0$ & 4.4 & $18 \cdot 6$ & 4.4 & 0.022 \\
\hline & Median & IQR & Median & IQR & Median & IQR & Median & IQR & Median & IQR & \\
\hline \multirow[t]{2}{*}{ Alcohol consumption (units/d)§ } & $1 \cdot 0$ & $0.4,2 \cdot 1$ & 0.9 & $0.4,1.7$ & 0.7 & $0.3,1.4$ & 0.7 & $0.3,1 \cdot 4$ & 0.6 & $0 \cdot 3,1 \cdot 3$ & $<0.001$ \\
\hline & $n$ & $\%$ & $n$ & $\%$ & $n$ & $\%$ & $n$ & $\%$ & $n$ & $\%$ & \\
\hline Women (\%) & 856 & $42 \cdot 0$ & 2022 & 51.6 & 1681 & $62 \cdot 0$ & 658 & 62.9 & 229 & $61 \cdot 2$ & $<0.001$ \\
\hline Marital status§ & & & & & & & & & & & 0.159 \\
\hline Single & 148 & $9 \cdot 9$ & 285 & 10 & 170 & $8 \cdot 8$ & 71 & $9 \cdot 8$ & 26 & $9 \cdot 7$ & \\
\hline Married & 1217 & 81.4 & 2322 & 81.4 & 1548 & $80 \cdot 3$ & 572 & $79 \cdot 2$ & 220 & 82.4 & \\
\hline Widowed/separated/divorced & 130 & $8 \cdot 7$ & 247 & $8 \cdot 7$ & 210 & $10 \cdot 9$ & 79 & $10 \cdot 9$ & 21 & $7 \cdot 9$ & \\
\hline Smoking status & & & & & & & & & & & $<0.001$ \\
\hline Never & 1042 & $51 \cdot 6$ & 2085 & $53 \cdot 7$ & 1516 & $56 \cdot 8$ & 582 & $56 \cdot 3$ & 183 & $49 \cdot 6$ & \\
\hline Ex-smoker & 667 & 33 & 1226 & 31.6 & 872 & $32 \cdot 7$ & 360 & $34 \cdot 9$ & 137 & $37 \cdot 1$ & \\
\hline Current & 310 & $15 \cdot 4$ & 573 & $14 \cdot 8$ & 283 & $10 \cdot 6$ & 91 & $8 \cdot 8$ & 49 & $13 \cdot 3$ & \\
\hline Under-reporter for EI $(\mathrm{El}: \mathrm{BMR}<1.4)$ & 1247 & $61 \cdot 1$ & 1913 & $48 \cdot 8$ & 1060 & $39 \cdot 1$ & 335 & 32 & 106 & $28 \cdot 3$ & $<0.001$ \\
\hline Intentional dieting (yes)§ & 111 & $7 \cdot 5$ & 202 & 7 & 176 & $8 \cdot 4$ & 73 & $8 \cdot 9$ & 32 & $10 \cdot 8$ & 0.068 \\
\hline Annual household income level & & & & & & & & & & & 0.083 \\
\hline$<£ 20000$ & 265 & $13 \cdot 5$ & 566 & $14 \cdot 9$ & 360 & $13 \cdot 6$ & 128 & $12 \cdot 5$ & 50 & $13 \cdot 7$ & \\
\hline$£ 20000-£ 40000$ & 679 & 34.5 & 1361 & $35 \cdot 8$ & 967 & $36 \cdot 6$ & 395 & $38 \cdot 7$ & 148 & $40 \cdot 8$ & \\
\hline$>£ 40000$ & 1026 & $52 \cdot 1$ & 1876 & $49 \cdot 3$ & 1314 & $49 \cdot 8$ & 498 & $48 \cdot 8$ & 165 & 45.5 & \\
\hline Occupational social class & & & & & & & & & & & 0.017 \\
\hline Routine and manual occupations & 339 & $17 \cdot 8$ & 648 & $17 \cdot 8$ & 410 & $16 \cdot 3$ & 147 & $15 \cdot 2$ & 67 & $19 \cdot 1$ & \\
\hline Intermediate occupations & 508 & $26 \cdot 6$ & 113 & 30.5 & 786 & $31 \cdot 3$ & 302 & $31 \cdot 2$ & 104 & 29.6 & \\
\hline Professional occupations & 1060 & $55 \cdot 6$ & 1884 & $51 \cdot 7$ & 1315 & $52 \cdot 4$ & 519 & $53 \cdot 6$ & 180 & $51 \cdot 3$ & \\
\hline Test site & & & & & & & & & & & 0.001 \\
\hline Cambridge & 729 & $35 \cdot 6$ & 1294 & 33 & 918 & 33.9 & 347 & $33 \cdot 1$ & 138 & $36 \cdot 9$ & \\
\hline Ely & 704 & 34.5 & 1475 & $37 \cdot 6$ & 1051 & $38 \cdot 8$ & 441 & $42 \cdot 1$ & 132 & $35 \cdot 3$ & \\
\hline Wisbech & 609 & 29.9 & 1152 & $29 \cdot 4$ & 741 & $27 \cdot 3$ & 259 & $24 \cdot 7$ & 104 & $27 \cdot 8$ & \\
\hline
\end{tabular}

El, energy intake.

* Snacking frequency was estimated using an eating pattern questionnaire reflecting usual eating habits over a 24-h period (see the 'Methods' section).

$\dagger$ ANOVA or a Kruskal-Wallis test for differences by frequency of snacking or $x^{2}$ test for heterogeneity.

‡ Total eating occasions = main meal + light meal + snack + drink-only snack.

$\S$ Due to some missing data, numbers do not always add to 10092 participants.

women who were not intentionally dieting were similar to those for all men and women (results not shown).

\section{Discussion}

In this large population-based study, we found that snacking frequency was inversely associated with measures of adiposity in normal-weight men and women, but was positively associated among the overweight or obese. Adjustment for overall dietary quality and PA did not affect these associations nor did adjusting for type of snack within the BMI groups. However, differences in the choice of snack between those who were normal weight and those who were overweight or obese informed the opposing direction of association by BMI status.

\section{Results in context}

To the best of our knowledge, this is the first study to examine snacking and adiposity measures other than BMI and to adjust for objectively measured PAEE and objectively measured fruit and vegetable intake as an indicator of dietary quality. Sex differences in the frequency of snacking and in the contribution of snacks to dietary intakes have been reported elsewhere ${ }^{(9,27)}$. In the present study, a higher frequency of snacking was associated with higher obesity (BMI) and body composition measures (waist circumference, subcutaneous abdominal fat and visceral fat thickness) in women but not among men. These observed sex differences were removed when we stratified by BMI status. After stratification, we found that snacking was 
Table 2. The association between snacking frequency* (per unit increase) and measures of adiposity by sex: the Fenland Study, UK ( $n$ 10 092) (Mean values and standard deviations; $\beta$ coefficients and $95 \%$ confidence intervals from multiple linear regression analysis)

\begin{tabular}{|c|c|c|c|c|c|c|c|c|}
\hline & \multirow[b]{2}{*}{ Mean } & \multirow[b]{2}{*}{ SD } & \multicolumn{3}{|c|}{ Model 1† } & \multicolumn{3}{|c|}{ Model $2 \ddagger$} \\
\hline & & & $\beta$ Coefficient & Lower $95 \% \mathrm{Cl}$ & Upper $95 \% \mathrm{Cl}$ & $\beta$ Coefficient & Lower $95 \% \mathrm{Cl}$ & Upper $95 \% \mathrm{Cl}$ \\
\hline \multicolumn{9}{|l|}{ Women } \\
\hline BMI $\left(\mathrm{kg} / \mathrm{m}^{2}\right)$ & $26 \cdot 5$ & $5 \cdot 3$ & 0.25 & 0.09 & 0.41 & 0.29 & 0.13 & 0.44 \\
\hline Waist circumference $(\mathrm{cm})$ & $85 \cdot 5$ & $12 \cdot 6$ & 0.7 & 0.32 & 1.08 & 0.73 & 0.4 & $1 \cdot 1$ \\
\hline Total body fat (\%) & 37.6 & $8 \cdot 1$ & 0.06 & -0.19 & 0.31 & $0 \cdot 12$ & -0.12 & 0.36 \\
\hline Visceral fat thickness $(\mathrm{cm})$ & $4 \cdot 3$ & 1.8 & 0.08 & 0.02 & $0 \cdot 13$ & 0.07 & 0.02 & 0.13 \\
\hline Subcutaneous abdominal fat thickness $(\mathrm{cm})$ & 3.2 & $1 \cdot 2$ & 0.04 & 0.01 & 0.08 & 0.05 & 0.02 & 0.08 \\
\hline \multicolumn{9}{|l|}{ Men } \\
\hline $\operatorname{BMI}\left(\mathrm{kg} / \mathrm{m}^{2}\right)$ & $27 \cdot 3$ & $4 \cdot 1$ & -0.06 & -0.18 & 0.07 & -0.02 & -0.15 & 0.1 \\
\hline Waist circumference $(\mathrm{cm})$ & $97 \cdot 2$ & 11.5 & -0.06 & -0.4 & $0 \cdot 28$ & 0.01 & -0.34 & 0.35 \\
\hline Total body fat (\%) & 27.6 & $7 \cdot 1$ & -0.26 & -0.48 & -0.04 & $-0 \cdot 1$ & -0.32 & 0.11 \\
\hline Visceral fat thickness $(\mathrm{cm})$ & $6 \cdot 3$ & $2 \cdot 1$ & 0.01 & -0.05 & 0.08 & 0.01 & -0.05 & 0.08 \\
\hline Subcutaneous abdominal fat thickness $(\mathrm{cm})$ & 2.5 & 1 & -0.02 & -0.05 & 0.01 & -0.01 & -0.05 & 0.02 \\
\hline
\end{tabular}

* Snacking frequency was estimated using an eating pattern questionnaire reflecting usual eating habits over a 24-h period. Energy intake was estimated using a FFQ (see the 'Methods' section).

† Model 1 adjusted for age (years), alcohol (units/d), smoking status (current smoker/non-smoker), age at completing full-time education (years), test site (Cambridge, Ely, Wisbech), main meal (frequency/d), light meal (frequency/d) and drink-only snack (frequency/d)

‡ Model 2: model 1 + plasma vitamin $\mathrm{C}(\mu \mathrm{mol} / \mathrm{l})$, energy intake $(\mathrm{MJ} / \mathrm{d})$, physical activity energy expenditure $(\mathrm{kJ} / \mathrm{kg}$ per $\mathrm{d})$ and screen time $(\mathrm{h})$

(1)

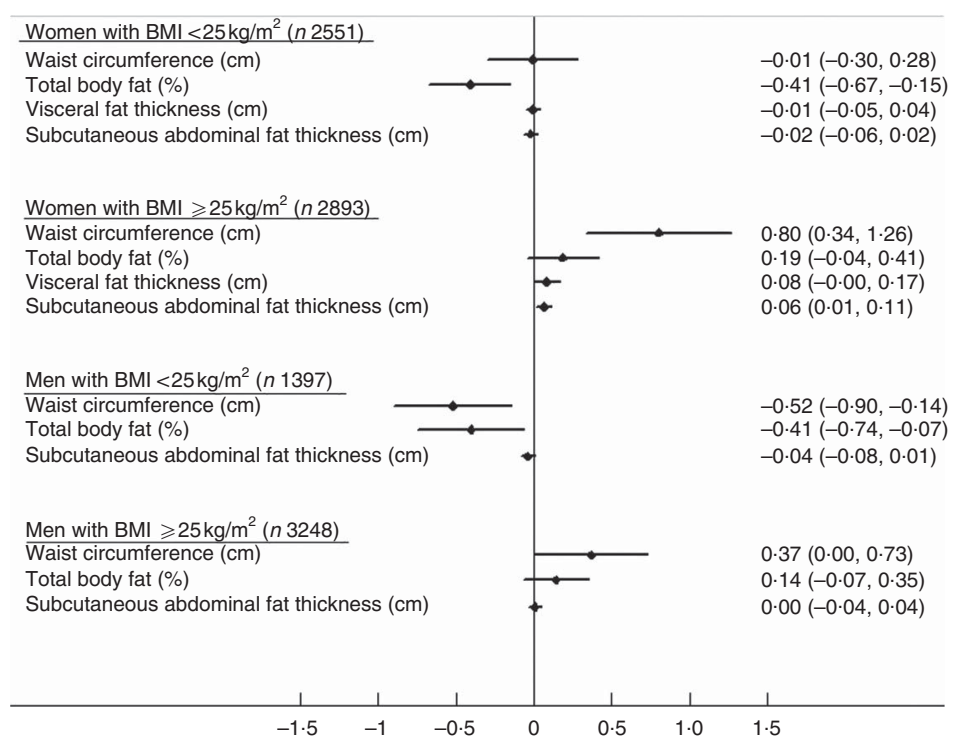

Fig. 1. The association between snacking frequency (per unit increase) and measures of adiposity by sex and BMI status: The Fenland Study, UK ( $n 10$ 092). Data are $\beta$-coefficients and $95 \%$ confidence intervals from multiple linear regression analysis. Comparison uses model 2, which is adjusted for age (years), alcohol (units/d), smoking status (current smoker/non-smoker), age at completing full-time education (years), test site (Cambridge, Ely, Wisbech), main meal (frequency/d), light meal (frequency/d), drink-only snack (frequency/d), plasma vitamin C ( $\mu \mathrm{mol} / \mathrm{l})$, energy intake $(\mathrm{MJ} / \mathrm{d})$, physical activity energy expenditure $(\mathrm{kJ} / \mathrm{kg}$ per d) and screen time (h). No interaction with BMI was noted between snacking and visceral fat thickness in men. Snacking frequency was estimated using an eating pattern questionnaire reflecting usual eating habit over a 24-h period. Energy intake was estimated using a FFQ (see the 'Methods' section).

inversely associated with total body fat in men and women of normal weight and with waist circumference in men of normal weight, but was positively associated with waist circumference and subcutaneous fat thickness in women who were overweight or obese. No significant associations between snacking frequency and visceral fat were apparent.

Snacking frequency has been associated cross-sectionally with increases in both 'healthy' and 'unhealthy' food choices, and different dietary patterns have been identified within high-frequency snacking groups ${ }^{(9)}$. Other evidences from cross-sectional studies have suggested a modest association between snacking and a more nutrient-dense diet ${ }^{(28)}$, and higher intakes of vitamins, carotenoids and minerals ${ }^{(29)}$. Snacking is also associated with eating more in general and choosing a wider variety of foods, resulting in a more balanced intake of nutrients ${ }^{(30)}$. The categorical term 'snacks' includes energy-dense and nutrient-poor foods, which are commonly referred to as snack foods, and also low energy-dense and high-fibre foods such as fruits. It is also generally thought that increased snacking is associated with lower PA levels, but the causal mechanism and the direction of the relationship remain uncertain. Bertéus Forslund et al. ${ }^{(27)}$ concluded that high PA 
Table 3. Snack-type food intakes* by BMI status: the Fenland Study, UK ( $n$ 10 092) (Mean values and standard deviations; medians and interquartile ranges (IQR))

\begin{tabular}{|c|c|c|c|c|c|}
\hline & \multicolumn{2}{|c|}{$\mathrm{BMl}<25 \mathrm{~kg} / \mathrm{m}^{2}$} & \multicolumn{2}{|c|}{$\mathrm{BMI} \geq 25 \mathrm{~kg} / \mathrm{m}^{2}$} & \multirow[b]{2}{*}{$P \dagger$} \\
\hline & Mean & SD & Mean & SD & \\
\hline Vegetables (g/10 MJ per d) & $246 \cdot 0$ & 248 & $247 \cdot 0$ & 203 & 0.846 \\
\hline \multirow[t]{2}{*}{ Fruit (g/10 MJ per d) } & $221 \cdot 0$ & 254 & $224 \cdot 0$ & 324 & 0.573 \\
\hline & Median & IQR & Median & IQR & \\
\hline Yoghurts (g/10 MJ per d) & $25 \cdot 0$ & $5 \cdot 3,66 \cdot 5$ & $22 \cdot 3$ & $0.0,65 \cdot 1$ & 0.021 \\
\hline Nuts $(g / 10 \mathrm{MJ}$ per $\mathrm{d})$ & 1.7 & $0.0,4.4$ & 1.6 & $0.0,3.6$ & $<0.001$ \\
\hline Crisps (g/10 MJ per $d)$ & $2 \cdot 6$ & $0 \cdot 6,10 \cdot 3$ & 3.3 & $1 \cdot 2,12 \cdot 4$ & $<0.001$ \\
\hline Cakes and biscuits ( $g / 10 \mathrm{MJ}$ per $d$ ) & $17 \cdot 0$ & $7 \cdot 0,37 \cdot 0$ & $16 \cdot 0$ & $6 \cdot 0,37 \cdot 0$ & 0.200 \\
\hline Chocolate (g/10 MJ per d) & 4.0 & $0.8,10.9$ & 4.5 & $1.0,15 \cdot 9$ & 0.001 \\
\hline Ice-creams (g/10 MJ per d) & 3.8 & $0.0,6.5$ & 4.2 & $0.0,7.4$ & $<0.001$ \\
\hline Sweets (g/10 MJ per $d)$ & 1.3 & $0.0,3.4$ & 1.4 & $0.0,3.9$ & $<0.001$ \\
\hline
\end{tabular}

* Snack-type food intakes were estimated using a FFQ (see the 'Methods' section). $\dagger$ The $t$ test or the Mann-Whitney $U$ test for difference.

could not explain high-energy intake and snacking, having found that energy intake was higher with higher snacking frequency, irrespective of PA level, but it has also been suggested that the effects of snacking on weight gain may be mediated by PA through increased energy requirements ${ }^{(31)}$. This disparity in the relationship between snacking frequency and dietary intakes and PA has been suggested as masking, and thus preventing the detection of an association between snacking and obesity or weight gain ${ }^{(5-8,30)}$. However, we found that accounting for the interaction with BMI status allowed for the detection of an association. There were different snack-type food intakes between the two groups, with those who were overweight or obese consuming less yoghurt and nuts and more ice-cream, sweets, chocolate and crisps compared with their normal-weight counterparts. One possible explanation for the different associations between BMI groups is differential gut hormone responses that different foods or nutrients may elicit. Gut hormones are known to both curb and increase appetite for example, ghrelin may increase appetite and oxyntomodulin and peptide $\mathrm{YY}_{3-36}$ may increase satiation ${ }^{(32)}$. In addition, a previous study has shown that healthy, non-obese adults may maintain a normal body weight by compensating for the consumption of snacks with increased PA or reduced energy intake at other meals ${ }^{(33)}$.

\section{Reporting bias}

The potential of intentional dieting contributing to the differential trends by BMI status was discussed at a symposium ${ }^{(34)}$ in 2012 and is supported in the present analysis as women with higher BMI reported lower energy intakes. Overall, our findings do not suggest that intentional dieting was driving the associations; however, we acknowledge that the number of individuals on a weight-loss diet in these analyses was small, and thus the effect of snacking among those on a diet may warrant further investigation.

As one of the major causes of dietary under-reporting is the failure to report foods eaten between meals ${ }^{(35)}$, reporting bias has the potential to create spurious results in any analysis of snacking. Consistent with this, we found that the percentage of energy under-reporters decreased as reported snacking frequency increased. However, the associations of snacking frequency with BMI and body composition remained similar after excluding potential under-reporters. This is in contrast with the eating frequency research, where the associations were attenuated to null when under-reporting was accounted for ${ }^{(4)}$. In addition, it is possible that participants associate snacking with weight gain, which may cause selective under-reporting of snacking frequency by those who are overweight or obese, thus causing the differential associations, although reported snacking frequencies did not differ by BMI status in the present study.

\section{Strengths and limitations}

The Fenland Study has a large population-based sample drawn from Cambridgeshire, which is representative of the general England population in terms of smoking and PA levels and somewhat healthier than the England average for obesity levels and healthy eating ${ }^{(36)}$. The strengths of the study are the use of many objective and precise measures of body composition, including DEXA and abdominal ultrasound, the inclusion of plasma vitamin $\mathrm{C}$ as an objectively measured marker of fruit and vegetable intake reflecting dietary quality and the inclusion of objectively measured PAEE. Our approach to analysis was rigorous, as we investigated possible under-reporting, intentional dieting and interaction by BMI and PAEE, allowing us to account for and exclude many alternative explanations for an association between snacking frequency and adiposity.

A further strength is that the present analysis may better reflect usual snacking behaviour of participants, capturing what they deem to be snacks. Although often defined as foods eaten between meals, there is no physiological basis to distinguish a meal from a snack ${ }^{(37)}$. A biologically based definition has been proposed: eating during a period of satiety rather than simply eating between meals ${ }^{(34)}$, while self-definition by participants has also been advocated ${ }^{(38)}$. In this study, participants were asked to describe their usual eating patterns for a 24-h period, giving information on the time of the day and the frequency of consumption of each meal type, whereas other studies have 
recorded snacking during a specific period of time or derived frequencies from food intake records where the researcher decides meal types.

This study has certain limitations. Its cross-sectional nature prevents the direction of the association from being determined, and we could not investigate the association of snacking with weight change. We were unable to validate the eating pattern (snacking frequency) questionnaire or examine the definition of a snack, drawing no distinction between eating between meals or eating in a state of satiety and relying on participants interpretation of what constitutes a snack. As eating pattern data (24-h eating pattern questionnaire) were collected separately from dietary intake data (FFQ), the energy and nutrient content of the snacks and which specific foods were eaten as snacks could not be determined. We did, however, make approximations using the FFQ data. We did not include drink-only snacks when estimating snacking frequency, as we considered snacking on foods and drinks to have different satiating effects; however, the analyses were adjusted for drink-only snack frequency. Although our assessment was comprehensive, data on eating patterns and dietary intake were self-reported and are subject to measurement error and bias that accompany such subjective assessment. We adjusted for a range of relevant potential confounding factors but residual confounding could not be ruled out.

\section{Conclusion}

In conclusion, snacking frequency was inversely associated with measures of adiposity in normal-weight men and women, but was positively associated in those who were overweight or obese. The differential association by BMI group may be due to differences in the choice of snack. The promotion of healthy snack choices could contribute to anti-obesity public health initiatives.

\section{Acknowledgements}

The authors are grateful to all the Fenland Study volunteers for their time and help and to the general practitioners and practice staff for help with recruitment. The authors thank the Functional groups of the MRC Epidemiology Unit, including the Fenland Study Co-ordination team, the Field Epidemiology team, the data management team and the laboratory team. The authors also thank Emanuella De Lucia Rolfe for anthropometry, DEXA and ultrasound data, and Kate Westgate and Stefanie Mayle for assistance with physical activity data processing. Biochemical assays were performed by the National Institute for Health Research, Cambridge Biomedical Research Centre, Core Biochemistry Assay Laboratory and the Cambridge University Hospitals NHS Foundation Trust, Department of Clinical Biochemistry.

The Fenland Study is funded by the Wellcome Trust and the Medical Research Council. Support from Medical Research Council programmes MC_UU_12015/1 and MC_UU_12015/5 is acknowledged.

The authors' responsibilities were as follows: L. O'C. performed the statistical analyses and drafted the manuscript.
N. G. F. and L. O'C. conceived the current research question, and N. G. F. provided input on the data analysis and interpretation of results. N. J. W., N. G. F., S. J. G. and S. B. are principal investigators of the Fenland Study and are responsible for the overall design and management of the study, with N. J. W. as chief investigator. All the authors contributed to the critical revisions and have read and approved the final version of the manuscript.

There are no conflicts of interest.

\section{References}

1. Piernas C \& Popkin BM (2010) Snacking increased among U.S. adults between 1977 and 2006. J Nutr 140, 325-332.

2. Kerr MA, Rennie KL, McCaffrey TA, et al. (2009) Snacking patterns among adolescents: a comparison of type, frequency and portion size between Britain in 1997 and Northern Ireland in 2005. Br J Nutr 101, 122-131.

3. Government Office for Science (2007) Foresight: tackling obesities: future choices - project report. https://www.gov. uk/government/uploads/system/uploads/attachment_data/file/ 287937/07-1184x-tackling-obesities-future-choices-report.pdf (accessed May 2015)

4. Bellisle F, McDevitt R \& Prentice AM (1997) Meal frequency and energy balance. Br J Nutr 77, S57-S70.

5. Howarth NC, Huang TTK, Roberts SB, et al. (2006) Eating patterns and dietary composition in relation to BMI in younger and older adults. Int J Obes 31, 675-684.

6. Bes-Rastrollo M, Sanchez-Villegas A, Basterra-Gortari FJ, et al. (2010) Prospective study of self-reported usual snacking and weight gain in a Mediterranean cohort: the SUN project. Clin Nutr 29, 323-330.

7. Hampl JS, Heaton CL \& Taylor CA (2003) Snacking patterns influence energy and nutrient intakes but not body mass index. J Hum Nutr Diet 16, 3-11.

8. Mills JP, Perry CD \& Reicks M (2011) Eating frequency is associated with energy intake but not obesity in midlife women. Obesity 19, 552-559.

9. Hartmann C, Siegrist M \& van der Horst K (2013) Snack frequency: associations with healthy and unhealthy food choices. Public Health Nutr 16, 1487-1496.

10. Pearson N \& Biddle SJ (2011) Sedentary behavior and dietary intake in children, adolescents, and adults. A systematic review. Am J Prev Med 41, 178-188.

11. Drewnowski A \& Bellisle F (2007) Liquid calories, sugar, and body weight. Am J Clin Nutr 85, 651-661.

12. Stolk RP, Wink O, Zelissen PM, et al. (2001) Validity and reproducibility of ultrasonography for the measurement of intra-abdominal adipose tissue. Int J Obes Relat Metab Disord 25, 1346-1351.

13. Rolfe Ede L, Loos RJ, Druet C, et al. (2010) Association between birth weight and visceral fat in adults. Am J Clin Nutr 92, 347-352.

14. Bingham SA, Welch AA, McTaggart A, et al. (2001) Nutritional methods in the European Prospective Investigation of Cancer in Norfolk. Public Health Nutr 4, 847-858.

15. Mulligan AA, Luben RN, Bhaniani A, et al. (2014) A new tool for converting food frequency questionnaire data into nutrient and food group values: FETA research methods and availability. BMJ Open $\mathbf{4}$, e004503.

16. Dehghan M, Akhtar-Danesh N, McMillan CR, et al. (2007) Is plasma vitamin $\mathrm{C}$ an appropriate biomarker of vitamin $\mathrm{C}$ intake? A systematic review and meta-analysis. Nutr J 6, 41. 
17. WHO/FAO (2003) Diet, Nutrition and the Prevention of Chronic Disease: Report of Joint WHO/FAO Expert Consultation. World Health Organization Technical Report Series, no. 196. Geneva, WHO.

18. Public Health England (2014) The Eatwell Plate. https://www.gov.uk/government/publications/the-eatwell-plateresources (accessed May 2015).

19. Brage S, Brage N, Franks PW, et al. (2005) Reliability and validity of the combined heart rate and movement sensor Actiheart. Eur J Clin Nutr 59, 561-570.

20. Brage S, Ekelund U, Brage N, et al. (2007) Hierarchy of individual calibration levels for heart rate and accelerometry to measure physical activity. J Appl Physiol 103, 682-692.

21. Stegle O, Fallert SV, MacKay DJ, et al. (2008) Gaussian process robust regression for noisy heart rate data. IEEE Trans Biomed Eng 55, 2143-2151.

22. Brage S, Brage N, Franks PW, et al. (2004) Branched equation modeling of simultaneous accelerometry and heart rate monitoring improves estimate of directly measured physical activity energy expenditure. $J$ Appl Physiol 96, 343-351.

23. Besson H, Brage S, Jakes RW, et al. (2010) Estimating physical activity energy expenditure, sedentary time, and physical activity intensity by self-report in adults. Am J Clin Nutr $\mathbf{9 1}$, 106-114.

24. Smith KJ, Blizzard L, McNaughton SA, et al. (2012) Daily eating frequency and cardiometabolic risk factors in young Australian adults: cross-sectional analyses. Br J Nutr 108, 1086-1094.

25. FAO/WHO/UNU (2001) Human Energy Requirements: Report of a Joint FAO/WHO/UNU Expert Consultation. Rome: FAO.

26. Goldberg GR, Black AE, Jebb SA, et al. (1991) Criticalevaluation of energy-intake data using fundamental principles of energy physiology: 1 . Derivation of cutoff limits to identify under-recording. Eur J Clin Nutr 45, 569-581.
27. Bertéus Forslund H, Torgerson JS, Sjostrom L, et al. (2005) Snacking frequency in relation to energy intake and food choices in obese men and women compared to a reference population. Int J Obes 29, 711-719.

28. Zizza CA \& Xu BB (2012) Snacking is associated with overall diet quality among adults. J Acad Nutr Diet 112, 291-296.

29. Zizza CA, Arsiwalla DD \& Ellison KJ (2010) Contribution of snacking to older adults' vitamin, carotenoid, and mineral intakes. J Am Diet Assoc 110, 768-772.

30. Whybrow S \& Kirk TR (1997) Nutrient intakes and snacking frequency in female students. J Hum Nutr Diet 10, 237-244.

31. Drummond S, Crombie N \& Kirk T (1996) A critique of the effects of snacking on body weight status. Eur J Clin Nutr $\mathbf{5 0}$, 779-783.

32. Murphy KG \& Bloom SR (2006) Gut hormones and the regulation of energy homeostasis. Nature 444, 854-859.

33. Viskaal-van Dongen M, Kok FJ \& de Graaf C (2010) Effects of snack consumption for 8 weeks on energy intake and body weight. Int J Obes 34, 319-326.

34. McCrory MA \& Campbell WW (2011) Effects of eating frequency, snacking, and breakfast skipping on energy regulation: symposium overview. J Nutr 141, 144-147.

35. Poppitt SD, Swann D, Black AE, et al. (1998) Assessment of selective under-reporting of food intake by both obese and non-obese women in a metabolic facility. Int $\mathrm{J}$ Obes Relat Metab Disord 22, 303-311.

36. Public Health England (2013) Health Profile Cambridgeshire 2012. http://www.apho.org.uk/resource/view.aspx?RID=117169 (accessed May 2015).

37. Chamontin AN, Pretzer G \& Booth DA (2003) Ambiguity of 'snack' in British usage. Appetite 41, 21-29.

38. Booth DA (1999) Evidence-based reduction of obesity: identification of a subculture's least fattening eating patterns. Appetite 32, 80-85. 